\title{
Stromal tumor-infiltrating lymphocytes evaluated on H\&E-stained slides are an independent prognostic factor in epithelial ovarian cancer and ovarian serous carcinoma
}

\author{
CHUNGSU HWANG ${ }^{1,2}$, SO JUNG LEE ${ }^{3}$, JUNG HEE LEE ${ }^{1,2}$, KI HYUNG KIM ${ }^{4}$, \\ DONG SOO $\mathrm{SUH}^{4}, \mathrm{BYUNG}^{-S U} \mathrm{KWON}^{4}$ and $\mathrm{KYUNG} \mathrm{UN} \mathrm{CHOI}^{3}$ \\ ${ }^{1}$ Department of Pathology, ${ }^{2}$ Research Institute for Convergence of Biomedical Science and Technology, \\ Pusan National University Yangsan Hospital, Yangsan, Gyeongsangnam-do 50612; Departments of ${ }^{3}$ Pathology, \\ and ${ }^{4}$ Obstetrics and Gynecology, Pusan National University Hospital, \\ Seo-Gu, Busan 602-739, Republic of Korea
}

Received September 13, 2018; Accepted February 1, 2019

DOI: $10.3892 / 01.2019 .10095$

\begin{abstract}
Studies on tumor-infiltrating lymphocytes (TILs) in epithelial ovarian cancer (EOC) have focused on the clinical significance of inflammatory cells of specific subtypes that are identifiable using immunohistochemistry. However, the subtypes of inflammatory cells that reportedly affect patient survival and prognosis have differed from study to study, and few studies have examined TILs using hematoxylin and eosin (H\&E) staining. Therefore, the present study aimed to identify the clinical importance of general stromal TILs in EOC by using H\&E staining to apply breast cancer recommendations from the International TILs Working Group 2014 on breast cancer using H\&E staining. Stromal TILs in 256 EOC cases from Pusan National University Hospital and 475 cases of high-grade serous carcinoma from The Cancer Genome Atlas dataset were assessed. Stromal TILs were evaluated using H\&E-stained slides according to the breast cancer recommendations of the International Working Group 2014, and patients were classified into low and high stromal TIL groups according to their stromal TIL values. The associations of these groups with clinicopathologic factors were assessed, and it was confirmed that group membership correlated
\end{abstract}

Correspondence to: Dr Kyung Un Choi, Department of Pathology, Pusan National University Hospital, 1-10 Ami-Dong, Seo-Gu, Busan 602-739, Republic of Korea

E-mail: kuchoi@pusan.ac.kr

Abbreviations: TIL, tumor-infiltrating lymphocyte; EOC, epithelial ovarian cancer; H\&E, hematoxylin and eosin; HGSC, high grade ovarian serous carcinoma; PNUH, Pusan National University Hospital; FIGO, International Federation of Gynecology and Obstetrics; TCGA, The Cancer Genome Atlas

Key words: ovarian cancer, tumor-infiltrating lymphocyte, survival, hematoxylin, eosin with survival and prognosis. According to the $\chi^{2}$ assessment, the stromal TIL group was associated with tumor grade. Furthermore, the stromal TIL group was associated with overall survival according to Kaplan-Meier analysis with the log-rank test. Finally, the stromal TIL group was an independent prognostic factor according to univariate and multivariate Cox regression analyses. In cases of EOC, the evaluation of general stromal TILs on H\&E-stained slides could be used to predict prognosis.

\section{Introduction}

Ovarian cancer is one of the leading causes of death in women. In 2018 alone, 14,070 women have died from this disease in United States (1). Although the standard treatment is cytoreductive surgery followed by chemotherapy, the mortality rate of ovarian cancer remains substantial and has not significantly improved. Thus, new treatments and methods of prognostic prediction need to be developed for women with ovarian cancer.

Recent studies have evaluated the importance of tumor-infiltrating lymphocytes (TILs) in various types of cancer, revealing that increased TIL levels are associated with better prognosis (2-5). Moreover, several studies have demonstrated that TILs are associated with the survival and prognosis of individuals with epithelial ovarian cancers (EOCs). Zhang et al found that a high counts of intratumoral $\mathrm{CD}^{+} \mathrm{T}$ cells were associated with higher progression-free and overall survival rates (6). Sato et al reported that high counts of intraepithelial $\mathrm{CD}^{+} \mathrm{T}$ cells, but not $\mathrm{CD} 3^{+} \mathrm{TILs}$, correlated with improved survival and prognosis (7). Webb et al revealed that intraepithelial CD103+ ${ }^{+}$TILs were strongly associated with patient survival, and affected the prognosis of high-grade ovarian serous carcinoma (HGSC) (8). Even though all of these studies used biomarkers to identify the subtypes of inflammatory cells that affect survival and prognosis, the biomarkers that were used and the results that were reported have not been consistent.

The International TILs Working Group 2014 on breast cancer provided recommendations for the evaluation of 
TILs on hematoxylin and eosin (H\&E)-stained slides. TILs assessed using these breast cancer recommendations have been applied as predictors of response to adjuvant or neoadjuvant chemotherapy and prognosis $(9,10)$. For ovarian cancers, TILs can also be assessed on H\&E-stained slides according to the recommendations of the International TILs Working Group 2014. However, the clinical significance of these TIL assessments remains to be determined. To date, most studies of ovarian cancers have focused only on TILs of specific subtypes located in the intraepithelial area, as evaluated using immunohistochemistry. Only one study has evaluated general TILs on H\&E-stained slides.

In the present study, we measured stromal TILs on H\&E-stained slides using the recommendations of the International TILs Working Group 2014 on breast cancer. Moreover, we evaluated the associations of stromal TILs with the survival and prognosis of individuals with EOCs.

\section{Materials and methods}

Patients. In total, 270 patients with primary EOCs who underwent explorative laparotomy at the Department of Gynecology of Pusan National University Hospital (PNUH) from 1998 to 2013 were included in the study. All patients provided written informed consent and underwent surgical procedures. We excluded patients who were not diagnosed with serous, mucinous, endometriod, and clear cell carcinoma, and analyzed cases with available tissue slides from the cohort of all patients. As a result, 256 patients were included in the study. The biospecimens for this study were provided by the Biobank of PNUH, a member of the National Biobank of Korea, which is supported by the Ministry of Health, Welfare and Family Affairs. All samples derived from the National Biobank of Korea were obtained with institutional review board approval.

All cases were examined by direct microscopic observation of H\&E-stained slides of formalin-fixed and paraffin-embedded surgical specimens. Tumor histology was identified according to the World Health Organization classification, and tumor stage was diagnosed based on the criteria of the International Federation of Gynecology and Obstetrics (FIGO). Other clinical data were obtained from the electronic medical records of PNUH. The mean age of patients was 53.5 years (range, 15-78 years). Clinicopathologic data, including tumor grade, mitosis, nuclear grade, tumor stage, histologic type, residual tumor, and chemotherapy response, are shown in detail in Table I. Tumor stage was reclassified as early stage for stage I and advanced stage for stages II, III, and IV. Overall survival was measured from diagnosis to death, and no patients were lost to follow-up.

Digital slides of high-grade ovarian serous carcinoma from The Cancer Genome Atlas (TCGA) dataset. The digital slide images of HGSCs were downloaded from the TCGA data portal (http://tcga-data.nci.nih.gov/). All digital slides were prepared from frozen specimens, and the digital slides of 475 patients were evaluated.

Evaluation method for stromal TILs. The stromal TILs were evaluated according to the recommendations of International TILs Working Group 2014 (11). The authors recommended
Table I. Clinicopathologic characteristics of the patients.

\begin{tabular}{|c|c|c|}
\hline Cliniopathologic characteristics & Numbers & Percentage, $\%$ \\
\hline \multicolumn{3}{|l|}{ Histologic types } \\
\hline Serous & 145 & 56 \\
\hline Mucinous & 48 & 19 \\
\hline Endometrioid & 20 & 8 \\
\hline Clear & 43 & 17 \\
\hline \multicolumn{3}{|l|}{ Tumor grade } \\
\hline 1 & 57 & 22 \\
\hline 2 & 127 & 50 \\
\hline 3 & 72 & 28 \\
\hline \multicolumn{3}{|l|}{ Nuclear grade } \\
\hline Mild & 17 & 7 \\
\hline Moderate & 125 & 49 \\
\hline Marked & 114 & 44 \\
\hline \multicolumn{3}{|l|}{ Mitosis } \\
\hline 0-9/10HPFs & 95 & 37 \\
\hline 10-24/10HPFs & 97 & 38 \\
\hline$\geq 25 / 10 \mathrm{HPFs}$ & 64 & 25 \\
\hline \multicolumn{3}{|l|}{ Tumor stage } \\
\hline Early stage & 72 & 38 \\
\hline Advanced stage & 118 & 62 \\
\hline \multicolumn{3}{|l|}{ Chemoresponse } \\
\hline Regressive disease & 77 & 44 \\
\hline Stable/progressive disease & 98 & 56 \\
\hline \multicolumn{3}{|l|}{ Residual tumor } \\
\hline Optimal & 173 & 91 \\
\hline Suboptimal & 18 & 9 \\
\hline
\end{tabular}

HPFs, high power fields (magnification, $\mathrm{x} 400$ ).

that TILs should be reported for the stromal compartment, with percentages, and evaluated within the tumor border. TILs in the tumor area with crush artifact, necrosis, or hyalinization should be excluded. Polymorphonuclear leukocytes should also bel excluded. Although one section per patient is sufficient for evaluation according to the recommendations, we assessed all available slides. The recommendations provided the table with detailed guidelines for assessing TILs, representative H\&E photographs, and illustrations for some TIL percentages, which were used to evaluate the stromal TILs (11). Two pathologists evaluated stromal TILs together. If their evaluations had different results, the final stromal TIL value was determined through consensus. Stromal TIL percentages were evaluated under a microscope via eye measurement. After evaluating the percentages of stromal TILs on all of the available H\&E-stained slides from the primary EOCs, the average stromal TIL percentage was calculated for each patient. This average stromal TIL percentage was used in the statistical analyses.

Statistical analysis. The $\chi^{2}$ test was used to assess relationships between stromal TILs and clinicopathologic factors. The 
Table II. Association between the stromal TIL group and clinicopathologic factors in all histologic types of epithelial ovarian cancer.

\begin{tabular}{|c|c|c|c|}
\hline \multirow[b]{2}{*}{ Variable } & \multicolumn{3}{|c|}{ Stromal TILs } \\
\hline & $\begin{array}{c}\text { Low } \\
\text { stromal } \\
\text { TILs }(\%)\end{array}$ & $\begin{array}{c}\text { High } \\
\text { stromal } \\
\text { TILs (\%) }\end{array}$ & P-value \\
\hline Residual tumor & & & 0.162 \\
\hline Optimal & $139(80)$ & $34(20)$ & \\
\hline Suboptimal & $13(72)$ & $5(23)$ & \\
\hline Tumor grade & & & 0.006 \\
\hline 1 & $51(89)$ & $6(11)$ & \\
\hline 2 & $105(83)$ & $22(17)$ & \\
\hline 3 & $49(68)$ & $23(32)$ & \\
\hline Histologic type & & & 0.002 \\
\hline Serous & $112(77)$ & $33(23)$ & \\
\hline Mucinous & 47 (98) & $1(2)$ & \\
\hline Endometrioid & $12(60)$ & $8(40)$ & \\
\hline Clear & $34(79)$ & $9(21)$ & \\
\hline Tumor stage & & & 1.000 \\
\hline Early stage & 57 (79) & $15(21)$ & \\
\hline Advanced stage & $94(80)$ & $23(20)$ & \\
\hline Nuclear grade & & & 0.032 \\
\hline Mild and moderate & $121(85)$ & $21(15)$ & \\
\hline Marked & $84(74)$ & $30(26)$ & \\
\hline Mitosis & & & 0.005 \\
\hline 0-9/10HPFs & $85(89)$ & $10(11)$ & \\
\hline 10-24/10HPFs & $76(78)$ & $21(22)$ & \\
\hline$\geq 25 / 10 \mathrm{HPFs}$ & $44(69)$ & $20(31)$ & \\
\hline Chemoresponse & & & 0.532 \\
\hline Regressive disease & $59(77)$ & $18(23)$ & \\
\hline Stable/progressive disease & $80(82)$ & $18(18)$ & \\
\hline
\end{tabular}

Bold numbers indicate $P<0.05$. TILs, tumor-infiltrating lymphocytes; HPFs, high power fields (magnification, $\mathrm{x} 400$ ).

overall survival rates of the low and high stromal TIL groups were analyzed using Kaplan-Meier survival analysis, and the statistical significance of between-group differences was evaluated using the log-rank test. Univariate and multivariate Cox regression analyses were performed to identify the prognostic significance of stromal TILs.

\section{Results}

Results of stromal TIL evaluations in the PNUH cohort. Among the patients who were evaluated, the number of H\&E-stained slides ranged from 1 to 15 , and the average number of slides per patient was 4.25 . The patients were classified into the high stromal TIL group if their stromal TIL percentages was $>10 \%$. They were classified into the low stromal TIL group if their stromal TIL percentage was similar
Table III. Association between the stromal TIL group and clinicopathologic factors in ovarian serous carcinomas.

\begin{tabular}{|c|c|c|c|}
\hline \multirow[b]{2}{*}{ Variable } & \multicolumn{2}{|c|}{ Stromal TILs } & \multirow[b]{2}{*}{ P-value } \\
\hline & $\begin{array}{c}\text { Low } \\
\text { stromal } \\
\text { TILs }(\%)\end{array}$ & $\begin{array}{c}\text { High } \\
\text { stromal } \\
\text { TILs }(\%)\end{array}$ & \\
\hline Residual tumor & & & 0.924 \\
\hline Optimal & $72(77)$ & $21(23)$ & \\
\hline Suboptimal & $10(83)$ & $2(17)$ & \\
\hline Tumor grade & & & 0.002 \\
\hline 1 and 2 & $80(86)$ & $13(14)$ & \\
\hline 3 & $32(62)$ & $20(38)$ & \\
\hline Simple stage & & & 0.596 \\
\hline Early stage & $15(71)$ & $6(29)$ & \\
\hline Advanced stage & $67(80)$ & $17(20)$ & \\
\hline Nuclear grade & & & 0.124 \\
\hline Mild and moderate & $60(83)$ & $12(17)$ & \\
\hline Marked & $52(71)$ & $21(29)$ & \\
\hline Mitosis & & & 0.006 \\
\hline 0-24/10 HPFs & $79(85)$ & $14(15)$ & \\
\hline$\geq 25 / 10$ HPFs & $33(63)$ & $19(37)$ & \\
\hline Chemoresponse & & & 0.539 \\
\hline Regressive disease & $34(76)$ & $11(24)$ & \\
\hline Stable/progressive disease & $43(83)$ & $9(17)$ & \\
\hline
\end{tabular}

Bold numbers indicate $\mathrm{P}<0.05$. TILs, tumor-infiltrating lymphocytes; HPFs, high power fields (magnification, x400).

to or $<10 \%$. Representative cases of the low and high stromal TIL groups are shown in Fig. 1.

The overall mean stromal TIL percentage was $6.94 \%$. As evaluated according to histologic type, the mean stromal TIL percentages for serous, mucinous, endometrioid, and clear cell carcinoma were 8.06,2.98, 8.66, and 6.75\%, respectively. Ovarian endometrioid carcinomas had the highest stromal TIL values, whereas ovarian mucinous carcinomas had the lowest values.

In total, 56 (22\%) of the 256 patients were included in the high stromal TIL group. Thirty-three (23\%) of the 145 patients with ovarian serous carcinomas were included in the high stromal TIL group. For the other histologic types of ovarian cancer, the percentages and counts of patients classified into the high stromal TIL group are presented in Table II.

To assess differences in stromal TILs across parts of the tumors, the standard deviations were calculated for each case and listed in the order of increasing means (Fig. 2). The result showed that the standard deviation and mean of the stromal TIL percentages increased together.

Results of stromal TIL evaluations using the TCGA dataset. The number of digital slides per patient ranged from 1 to 4 , and the average number of digital slides per patient was 1.96. The patients were also classified into the high and low stromal TIL groups based on the same criteria that were applied for the 

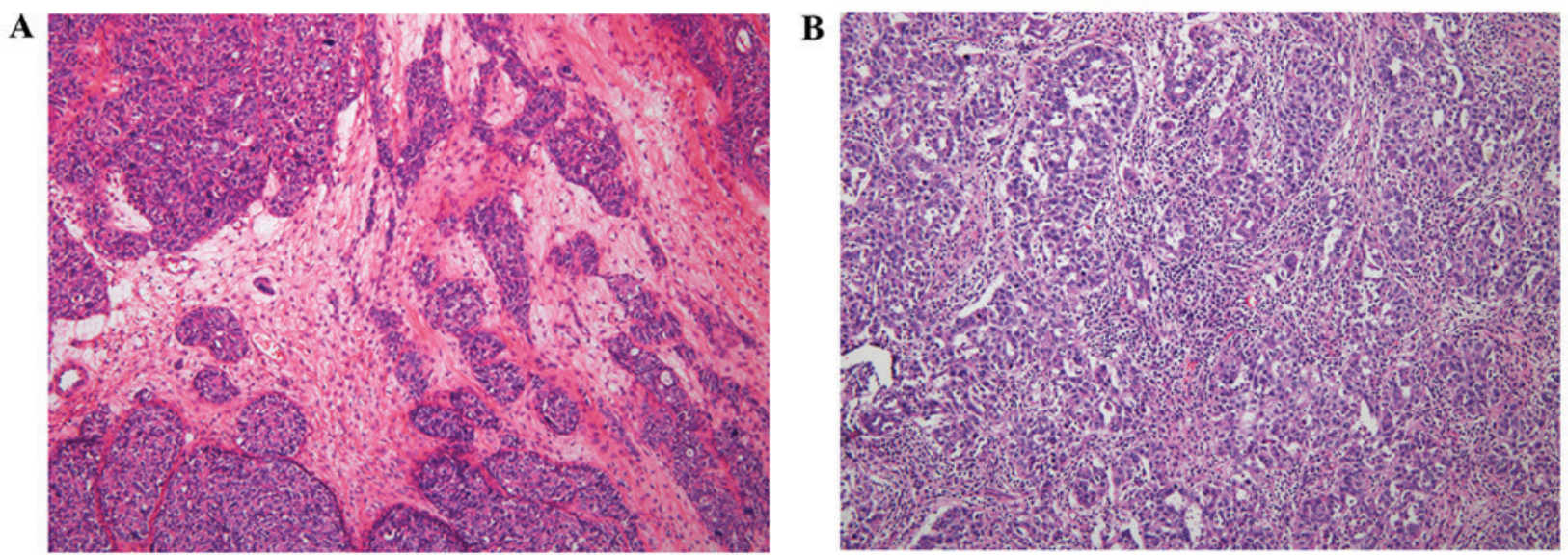

Figure 1. (A) Representative case of low stromal TIL group. Few inflammatory cells were identified in the stroma (magnification, x100). (B) Representative case of high stromal TIL group. Almost all stroma was covered with inflammatory cells (magnification, x100). TIL, tumor-infiltrating lymphocyte.
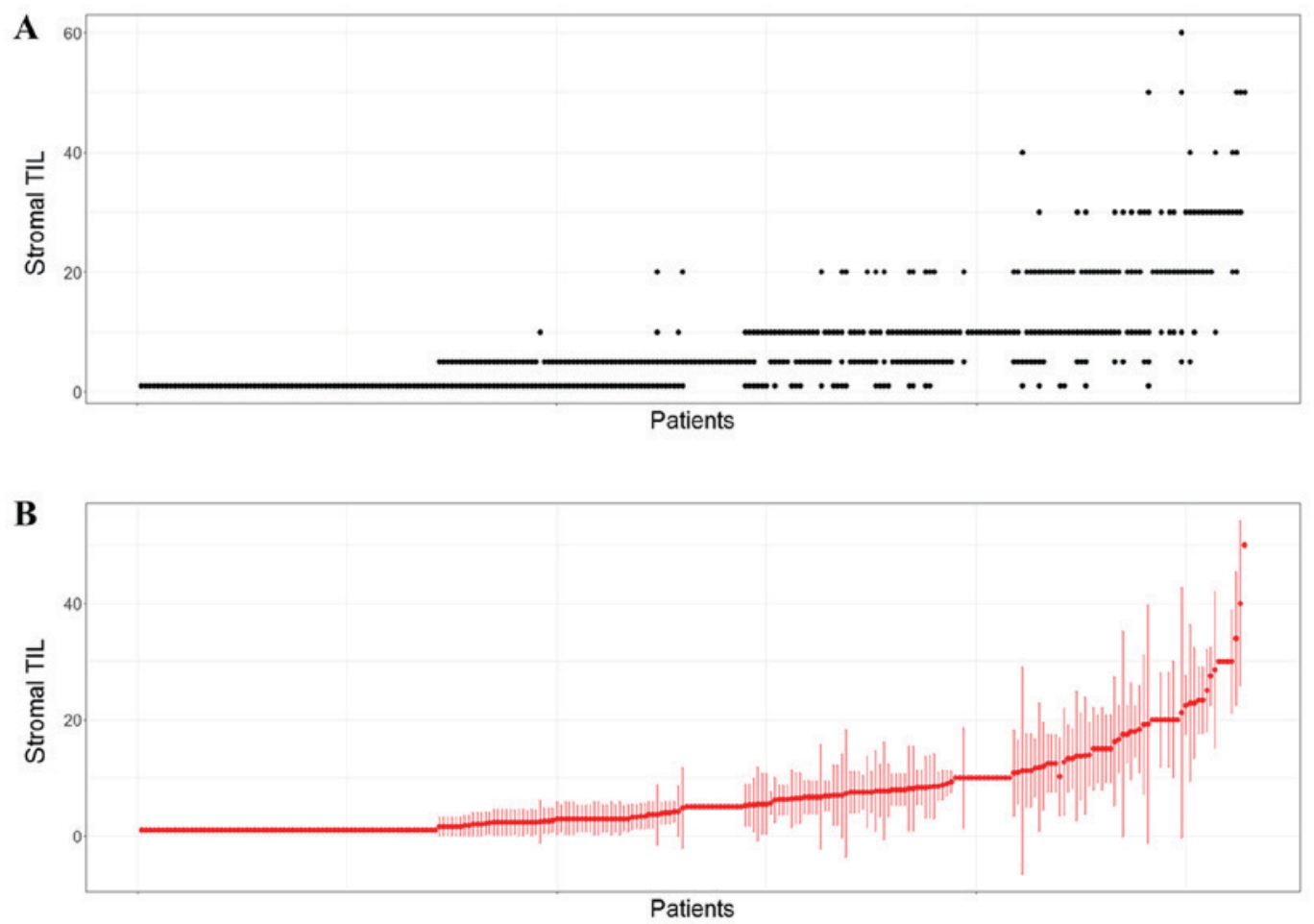

Figure 2. (A) Stromal TIL values of each patient. Black dots indicated the stromal TIL values of each slide. (B) Mean and standard deviation for each patient. Red dots and red bars indicated mean values and standard deviations of stromal TIL of each patient, respectively. TIL, tumor-infiltrating lymphocyte.

PNUH cohort. In the TCGA dataset, the average percentage of stromal TILs was $11.49 \%$, and 164 (35\%) of 475 patients were included in the high stromal TIL group.

Associations between stromal TILs and clinicopathologic characteristics in the PNUH cohort and TCGA dataset. The chi-square test revealed that tumor grade, histologic type, nuclear grade, and mitosis were associated with stromal TILs (Table II). Higher tumor grade, nuclear grade, and mitosis were each associated with higher frequencies of stromal TIL cases.

Ovarian serous carcinoma is the most common type of EOC. Thus, it has been the focus of the present study. In serous carcinoma, stromal TILs were associated with tumor grade and mitosis. Higher tumor grade and mitosis were each associated with higher frequencies of stromal TIL cases (Table III).

For high-grade serous ovarian cancer cases from the TCGA dataset, none of the investigated clinicopathologic factors (size of residual tumor, tumor stage, tumor grade primary therapy outcome, or platinum status) were associated with stromal TILs.

Survival analysis of the PNUH cohort. Kaplan-Meier survival analysis and the log-rank test revealed that the high stromal TIL group had a higher overall survival rate than the low stromal TIL group ( $\mathrm{P}=0.001)$ (Fig. 3A). We performed univariate and multivariate analyses of the pathologic prognostic factors, including tumor grade, tumor stage, residual tumor after 
Table IV. Univariate and multivariate Cox regression analysis in all histologic types of epithelial ovarian cancer.

\begin{tabular}{|c|c|c|c|c|}
\hline \multirow[b]{2}{*}{ Variable } & \multicolumn{2}{|c|}{ Univariate } & \multicolumn{2}{|c|}{ Multivariate } \\
\hline & HR (95\% CI for HR) & P-value & HR (95\% CI for HR) & P-value \\
\hline \multicolumn{5}{|l|}{ Stromal TILs } \\
\hline Low group & 1 [Reference] & & 1 [Reference] & \\
\hline High group & $0.379(0.203-0.707)$ & 0.002 & $0.343(0.17-0.691)$ & 0.003 \\
\hline \multicolumn{5}{|l|}{ Residual tumor } \\
\hline Optimal & 1 [Reference] & & 1 [Reference] & \\
\hline Suboptimal & $2.551(1.377-4.723)$ & 0.003 & $1.687(0.903-3.15)$ & 0.101 \\
\hline \multicolumn{5}{|l|}{ Tumor grade } \\
\hline Grade 1 & 1 [Reference] & & 1 [Reference] & \\
\hline Grade 2 & $3.685(1.895-7.168)$ & $<0.001$ & $2.119(0.929-4.836)$ & 0.074 \\
\hline Grade 3 & $3.517(1.738-7.119)$ & $<0.001$ & $2.753(1.158-6.546)$ & 0.022 \\
\hline \multicolumn{5}{|l|}{ Simple stage } \\
\hline Early stage & 1 [Reference] & & 1 [Reference] & \\
\hline Advanced stage & $5.598(2.956-10.6)$ & $<0.001$ & $4.303(2.207-8.389)$ & $<0.001$ \\
\hline
\end{tabular}

Bold numbers indicate $\mathrm{P}<0.05$. TILs, tumor-infiltrating lymphocytes; HR, hazard ratio; CI, confidence interval.

Table V. Univariate and multivariate Cox regression analysis in ovarian serous carcinomas.

\begin{tabular}{|c|c|c|c|c|}
\hline \multirow[b]{2}{*}{ Variable } & \multicolumn{2}{|c|}{ Univariate } & \multicolumn{2}{|c|}{ Multivariate } \\
\hline & HR (95\% CI for HR) & P-value & HR (95\% CI for HR) & $\mathrm{P}$-value \\
\hline \multicolumn{5}{|l|}{ Stromal TILs } \\
\hline Low group & 1 [Reference] & & 1 [Reference] & \\
\hline High group & $0.218(0.087-0.543)$ & 0.001 & $0.255(0.09-0.72)$ & 0.010 \\
\hline \multicolumn{5}{|l|}{ Residual tumor } \\
\hline Optimal & 1 [Reference] & & 1 [Reference] & \\
\hline Suboptimal & $2.458(1.143-5.283)$ & 0.021 & $1.638(0.759-3.533)$ & 0.208 \\
\hline \multicolumn{5}{|l|}{ Tumor grade } \\
\hline Grade 1 & 1 [Reference] & & 1 [Reference] & \\
\hline Grade 2 & $8.337(1.148-60.52)$ & 0.036 & $3.35(0.451-24.881)$ & 0.237 \\
\hline Grade 3 & $6.077(0.817-45.21)$ & 0.078 & $3.424(0.446-26.29)$ & 0.237 \\
\hline \multicolumn{5}{|l|}{ Tumor stage } \\
\hline Early stage & 1 [Reference] & & 1 [Reference] & \\
\hline Advanced stage & $17.19(2.365-124.9)$ & 0.005 & $13.21(1.976-97.1)$ & 0.011 \\
\hline
\end{tabular}

Bold numbers indicate $\mathrm{P}<0.05$. TILs, tumor-infiltrating lymphocytes; HR, hazard ratio; CI, confidence interval.

surgery, and stromal TILs. Results showed that stromal TIL, tumor grade 3 , and tumor stage were independent prognostic factors (Table IV).

For patients with ovarian serous carcinoma, stromal TILs were significantly associated with the overall survival rate $(\mathrm{P}<0.001)$ (Fig. 3B). Univariate and multivariate analyses showed that stromal TILs and tumor stage were independent prognostic factors (Table V).

Stromal TIL was significantly associated with overall survival in individuals with ovarian mucinous carcinoma, according to Kaplan-Meier survival analysis and the log-rank test $(\mathrm{P}=0.029)$. However, this result was not reliable because only one case was included in the high stromal TIL group. The associations between stromal TILs and the overall survival rates of individuals with endometrioid and clear cell carcinoma were not statistically significant $(\mathrm{P}=0.241$ and 0.317 , respectively).

Survival analysis using the TCGA dataset. Kaplan-Meier survival analysis and the log-rank test revealed that the 
Table VI. Univariate and multivariate cox regression analysis for overall survival in high-grade ovarian serous carcinoma of The Cancer Genome Atlas dataset.

Univariate

Variable
HR (95\% CI for HR)

1 [Reference]

$0.744(0.57-0.971)$

1 [Reference]

$2.399(1.128-5.102)$

$3.153(1.425-6.977)$

1 [Reference]

$1.325(0.917-1.915)$

1 [Reference]

$1.33(1.014-1.742)$
0.029

1 [Reference]

0.764 (0.576-1.014)

0.063

1 [Reference]

0.023

2.535 (1.023-6.285)

$3.094(1.202-7.965)$

0.045

0.019

1 [Reference]

1.344 (0.904-1.999)

1 [Reference]

1.263 (0.955-1.67)

0.102
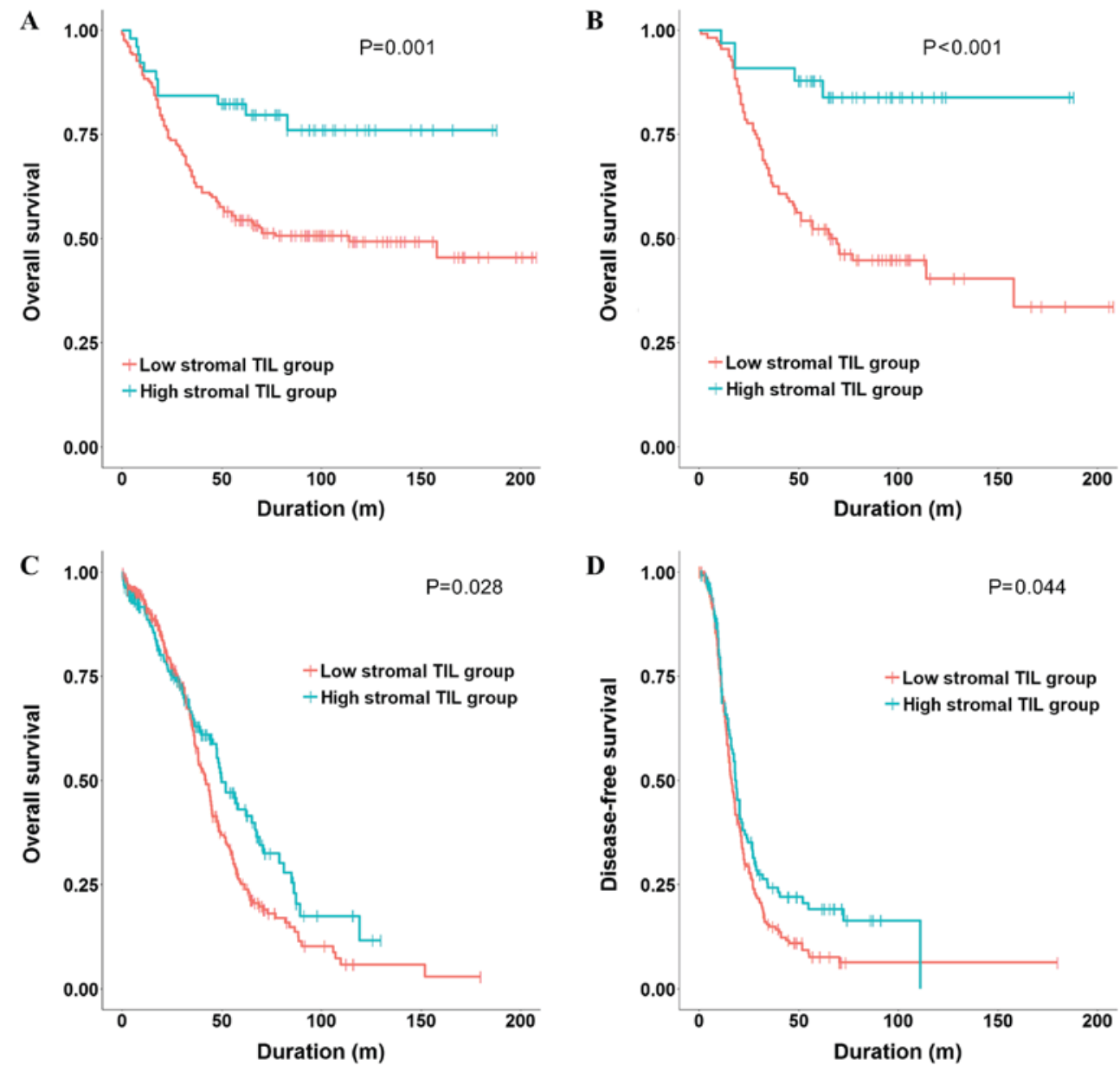

Figure 3. Kaplan-Meier curve analysis and log rank test. (A) Kaplan-Meier curve for overall survival according to the stromal TIL groups in all histologic types of EOCs from PNUH. (B) Kaplan-Meier curve for overall survival according to the stromal TIL groups in ovarian serous carcinoma from PNUH. (C) Kaplan-Meier curve for overall survival according to the stromal TIL groups in HGSC from TCGA dataset. (D) Kaplan-Meier curve for disease-free survival according to the stromal TIL groups in HGSC from TCGA dataset. TIL, tumor-infiltrating lymphocyte. epithelial ovarian cancer; EOCs, epithelial ovarian cancers; PNUH, Pusan National University Hospital; HGSC, high-grade ovarian serous carcinoma; TCGA, The Cancer Genome Atlas. 
Table VII. The result of univariate and multivariate cox regression analysis for disease-free survival in HGSC of TCGA dataset.

\begin{tabular}{|c|c|c|c|c|}
\hline \multirow[b]{2}{*}{ Variable } & \multicolumn{2}{|c|}{ Univariate } & \multicolumn{2}{|c|}{ Multivariate } \\
\hline & HR (95\% CI for HR) & P-value & HR (95\% CI for HR) & P-value \\
\hline \multicolumn{5}{|l|}{ Stromal TIL } \\
\hline Low & 1 [Reference] & & 1 [Reference] & \\
\hline High & $0.77(0.597-0.994)$ & 0.044 & $0.748(0.568-0.985)$ & 0.039 \\
\hline \multicolumn{5}{|l|}{ Tumor stage } \\
\hline Stage II & 1 [Reference] & & 1 [Reference] & \\
\hline Stage III & $1.895(1.103-3.257)$ & 0.021 & $1.812(0.963-3.411)$ & 0.066 \\
\hline Stage IV & $2.475(1.351-4.534)$ & 0.003 & $2.451(1.223-4.914)$ & 0.012 \\
\hline \multicolumn{5}{|l|}{ Tumor grade } \\
\hline Grade 2 & 1 [Reference] & & 1 [Reference] & \\
\hline Grade 3 & $1.329(0.938-1.882)$ & 0.109 & $1.393(0.939-2.064)$ & 0.099 \\
\hline \multicolumn{5}{|c|}{ Residual tumor } \\
\hline Optimal & 1 [Reference] & & 1 [Reference] & \\
\hline Suboptimal & $1.164(0.881-1.537)$ & 0.285 & $1.13(0.851-1.5)$ & 0.398 \\
\hline
\end{tabular}

Bold numbers indicate $\mathrm{P}<0.05$.

high stromal TIL group had significantly better overall and disease-free survival rates than the low stromal TIL group ( $\mathrm{P}=0.028$ and 0.044 , respectively) (Fig. 3C and D, respectively). We performed Cox regression analyses of the pathologic prognostic factors, including tumor stage, tumor grade, residual tumor, and stromal TILs. In the univariate analysis of the overall survival rate, stromal TILs, tumor stage, and residual tumor were considered as prognostic factors, and stromal TIL was a favorable prognostic factor (Table VI). Tumor stage remained a prognostic factor in the multivariate Cox regression analysis of the overall survival rate. However, stromal TIL and residual tumor were not prognostic factors in the multivariate analysis.

In the univariate and multivariate Cox regression analyses of the disease-free survival rate, stromal TIL was a statistically independent and favorable prognostic factor (Table VII).

\section{Discussion}

Several previous studies have evaluated TILs on H\&E-stained slides using a method suggested by the International TILs Working Group 2014 on breast, lung, stomach, and esophageal cancer. The results of these studies showed that stromal TILs were associated with survival rates and had prognostic effects $(9,12-17)$. The results were particular striking for breast cancer, stromal TIL was not only a prognostic factor, but also a predictor of responses to both adjuvant and neoadjuvant chemotherapy in certain types of breast cancer. For example, Loi et al reported that an increase in stromal TILs was associated with benefits of anthracycline-only chemotherapy in individuals with HER2-positive breast cancer (9). Additionally, Herrero-Vicent et al demonstrated that the rate of pathologic complete remission of tumor and lymphadenopathy was higher in individuals with lymphocyte-predominant triple-negative breast cancer when neoadjuvant chemotherapy was performed before surgery (10). Furthermore, it is known that stromal TIL is more stable and reproducible than intratumoral TIL in breast cancer (18). These results suggested that TILs assessed on $\mathrm{H} \& \mathrm{E}$-stained slides can be used as a predictor of prognosis or therapeutic response. The results also suggested that it would be worth investigating whether TILs assessed using H\&E-stained slides were clinically significant in ovarian cancers.

Nevertheless, only one study of EOC has evaluated TILs with H\&E-stained slides using the method suggested by an International TILs Working Group 2014 on breast cancer (19). To date, most studies of EOC have instead evaluated TILs using immunostaining for immune-related biomarkers, such as CD3, CD8, or FoxP3 (7,20,21). James et al (19) indicated that the findings of studies that used immunostaining differed in terms of immune-related biomarkers that affect prognosis. These results suggested that the evaluation of all types of inflammatory cells on H\&E-stained slides would be more effective in confirming tumor immunity than in assessing TILs of a particular subtype. Therefore, we evaluated stromal TILs on H\&E-stained slides using a method suggested by the International TIL Working Group 2014 on breast cancer and identified their clinical significance.

Based the standard deviations of stromal TILs that were observed in the present study, the evaluation of as many tumor slides as possible may help to improve the accuracy of stromal TIL measurements in cases of EOC. However, the International TIL Working Group 2014 recommendations showed that the evaluation of only one representative slide is sufficient. EOCs generally have ambiguous boundaries with normal tissue, and they are larger than breast cancers. In addition, more intratumoral changes, such as necrosis, are observed. Therefore, in EOCs, evaluating as many slides as possible may improve the accuracy of stromal TIL measurements.

Our study showed that stromal TIL was an independent prognostic factor for EOC overall (all histologic types) and for ovarian serous carcinoma, specifically. James et al (19) also 
evaluated TILs on H\&E-stained slides and showed that stromal TILs were not an independent prognostic factor for EOCs, which is contrary to our results. Our study and the study of James et al both followed the standardized method described by Salgado et al (11) for breast cancer, and both studies assessed TILs via eye measurement. The proportion of cases with stromal TIL values above the cutoff value of $10 \%$ was approximately the same in both studies, which indicates that there is no difference in stromal TIL readings. In the previous study, James et al evaluated a large number of cases and performed survival analysis by dividing the cases into three groups according to TILs, and this is the only difference from the present study.

Although the cytotoxic T lymphocyte-mediated direct killing mechanism is an important part of $\mathrm{T}$ cell-mediated cancer regression, $\mathrm{T}$ cells exposed to tumor antigens must first migrate to the tumor site. To overcome the various mechanisms of immune invasion by cancer cells, it is necessary that as many $\mathrm{T}$ cells as possible accumulate at the tumor site (22). Schietinger et al (23) showed that T cell-induced destruction of stromal components, including blood vessels, leads to cancer necrosis, and this phenomenon also occurs in individuals with antigen-negative cancer cell variants. These results suggest that the accumulation of TILs in the stroma without direct interaction with cancer cells is extremely important for cancer removal and that stromal TILs can influence the clinical outcomes.

In conclusion, we evaluated stromal TILs on H\&E-stained slides using a method suggested by the International TILs Working Group 2014 on breast cancer. We found that this evaluation of stromal TILs could be useful for predicting the prognosis of patients with EOC, particularly those with serous ovarian cancers. More studies should be performed to evaluate the precise clinical significance of stromal TILs assessed on H\&E-stained slides.

\section{Acknowledgements}

Not applicable.

\section{Funding}

This study was supported by a 2-Year Research Grant of Pusan National University.

\section{Availability of data and materials}

The datasets used during the present study are available from the corresponding author upon reasonable request.

\section{Authors' contributions}

$\mathrm{CH}$ and KUC designed the experiments and wrote the manuscript. KHK, DSS and BSK contributed to the acquisition of data and approved the final version of the version to be published. CH, SJL and JHL analyzed the data. All authors read and approved the final manuscript.

\section{Ethics approval and consent to participate}

This study was approved by the ethics committee of Pusan National University Hospital (Busan, South Korea) and informed consent was obtained from all individual participants included in the study.

\section{Patient consent for publication}

Consent was obtained from all individual participants included in the study.

\section{Competing interests}

The authors declare that they have no competing interests.

\section{References}

1. Seigel RL, Miller KD and Jemal A: Cancer statistics, 2018. CA Cancer J Clin 68: 7-30, 2018

2. Clemente CG, Mihm MC Jr, Bufalino R, Zurrida S, Collini P and Cascinelli N: Prognostic value of tumor infiltrating lymphocytes in the vertical growth phase of primary cutaneous melanoma. Cancer 77: 1303-1310, 1996.

3. Mahmoud SM, Paish EC, Powe DG, Macmillan RD, Grainge MJ, Lee AH, Ellis IO and Green AR: Tumor-infiltrating $\mathrm{CD} 8^{+}$lymphocytes predict clinical outcome in breast cancer. J Clin Oncol 29: 1949-1955, 2011.

4. Ropponen KM, Eskelinen MJ, Lipponen PK, Alhava E and Kosma VM: Prognostic value of tumour-infiltrating lymphocytes (TILs) in colorectal cancer. J Pathol 182: 318-324, 1997.

5. Fukunaga A, Miyamoto M, Cho Y, Murakami S, Kawarada Y, Oshikiri T, Kato K, Kurokawa T, Suzuoki M, Nakakubo Y, et al: $\mathrm{CD}^{+}$tumor-infiltrating lymphocytes together with $\mathrm{CD}^{+}+$tumor-infiltrating lymphocytes and dendritic cells improve the prognosis of patients with pancreatic adenocarcinoma. Pancreas 28: e26-e31, 2004.

6. Zhang L, Conejo-Garcia JR, Katsaros D, Gimotty PA, Massobrio M, Regnani G, Makrigiannakis A, Gray H, Schlienger K, Liebman MN, et al: Intratumoral T cells, recurrence, and survival in epithelial ovarian cancer. N Engl J Med 348:203-213, 2003.

7. Sato E, Oison SH, Ahn J, Bundy B, Nishikawa H, Qian F, Jungbluth AA, Frosina D, Gnjatic S, Ambrosone C, et al: Intraepithelial $\mathrm{CD} 8^{+}$tumor-infiltrating lymphocytes and a high $\mathrm{CD} 8^{+} /$regulatory $\mathrm{T}$ cell ratio are associated with favorable prognosis in ovarian cancer. Proc Natl Acad Sci USA 102: 18538-18543, 2005.

8. Webb JR, Milne K, Watson P, Deleeuw RJ and Nelson BH. Tumor-infiltrating lymphocytes expressing the tissue resident memory marker CD103 are associated with increased survival in high-grade serous ovarian cancer. Clin Cancer Res 20: 434-444, 2014.

9. Loi S, Sirtaine N,Piette F, Salgado R, Viale G, Van Eenoo F, Rouas G, Francis P, Crown JP, Hitre E, et al: Prognostic and predictive value of tumor-infiltrating lymphocytes in a phase III randomized adjuvant breast cancer trial in node-positive breast cancer comparing the addition of docetaxel to doxorubicin with doxorubicin-based chemotherapy: BIG 02-98. J Clin Oncol 31: 860-867, 2013.

10. Herrero-Vicent C, Guerrero A, Gavila J, Gozalbo F, Hernandez A, Sandiego S, Algarra MA, Calatrava A, Guillem-Porta V and Ruiz-Simon A: Predictive and prognostic impact of tumour-infiltrating lymphocytes in triple-negative breast cancer treated with neoadjuvant chemotherapy. Ecancermedicalscience 11: 759, 2017.

11. Salgado R, Denkert C, Demaria S, Sirtaine N, Klauschen F, Pruneri G, Wienert S, Van den Eynden G, Baehner FL, Penault-Llorca F, et al: The evaluation of tumor-infiltrating lymphocytes (TILs) in breast cancer: recommendations by an International TILs Working Group 2014. Ann Oncol 26: 259-271, 2014.

12. Loi S, Michiels S, Salgado R, Sirtaine N, Jose V, Fumagali D, Kellokumpu-Lehtinen PL, Bono P, Kataja V, Desmedt C, et al: Tumor infiltrating lymphocytes are prognostic in triple negative breast cancer and predictive for trastuzumab benefit in early breast cancer: Results from the FinHER trial. Ann Oncol 25: 1544-1550, 2014.

13. Denkert C, von Minckwitz G, Brase JC, Sinn BV, Gade S, Kronenwett R, Pfitzner BM, Salat C, Loi S, Schmitt WD, et al: Tumor-infiltrating lymphocytes and response to neoadjuvant chemotherapy with or without carboplatin in human epidermal growth factor receptor 2-positive and triple-negative breast cancers. J Clin Oncol 33: 983-991, 2015. 
14. Adams S, Gray RJ, Demaria S, Goldstein L, Perez EA, Shulman LN, Martino S, Wang M, Jones VE, Saphener TJ, et al: Prognostic value of tumor-infiltrating lymphocytes in triple-negative breast cancers from two phase III randomized adjuvant breast cancer trials: ECOG 2197 and ECOG 1199. J Clin Oncol 32: 2959-2966, 2014.

15. Mignon S, Wilard-Gallo K, Van den Eynden G, Salgado R, Waelput W, Decoster L, Marien K, Vansteenkiste J, Teugels E and De Greve J: The relationship of TILs and PD-L1 expression in NSCLC adenocarcinoma in little to non-smokers with driver mutations and outcome parameters. J Thorac Oncol 12: S1331, 2017.

16. Kang BW, Seo An, Yoon S, Bae HI, Jeon SW, Kwon OK, Chung HY, Yi W, Kang H and Kim JG: Prognostic value of tumor-infiltrating lymphocytes in Epstein-Barr virus-associated gastric cancer. Ann Oncol 27: 494-501, 2016.

17. Sudo T, Nishida R, Kawahara A, Saisho K, Mimori K, Yamada A, Mizogchi A, Kadoya K, Matono S, Mori N, et al: Clinical impact of tumor-infiltrating lymphocytes in esophageal squamous cell carcinoma. Ann Surg Oncol 24: 3763-3770, 2017.

18. Ahn SG, Jeong J, Hong S and Jung WH. Current issues and clinical evidence in tumor-infiltrating lymphocytes in breast cancer. J Pathol Transl Med 49: 355-363, 2015.
19. James FR, Jiminez-Linan M, Alsop J, Mack M, Song H, Brenton JD, Pharoah PDP and Ali HR: Association between tumor infiltrating lymphocytes, histotype and clinical outcome in epithelial ovarian cancer. BMC Cancer 17: 657, 2017.

20. Tomsová M, Melichar B, Sedlákovál I and Steiner I: Prognostic significance of $\mathrm{CD}^{+}$tumor-infiltrating lymphocytes in ovarian carcinoma. Gynecol Oncol 108: 415-420, 2008.

21. Hermans C, Anz D, Engel J, Kirhner T, Endres S and Mayr D: Analysis of FoxP3 ${ }^{+}$T-regulatory cells and $\mathrm{CD} 8^{+} \mathrm{T}$-cells in ovarian carcinoma: Location and tumor infiltrating patterns are key prognostic markers. PLos One 9: e111757, 2014.

22. Slaney CY, Kershaw MH and Darcy PK: Trafficking of T cells into tumor. Cancer Res 74: 7168-7174, 2014

23. Schietinger A, Arina A, Liu RB, Wells S, Huang J, Engels B, Bindokas V, Bartkowiak T, Lee D, Hermann A, et al: Longitudinal confocal microscopy imaging of solid tumor destruction following adoptive T cell transfer. Oncoimmunology 2: e26677, 2013.

This work is licensed under a Creative Commons

Attribution-NonCommercial-NoDerivatives 4.0 International (CC BY-NC-ND 4.0) License. 